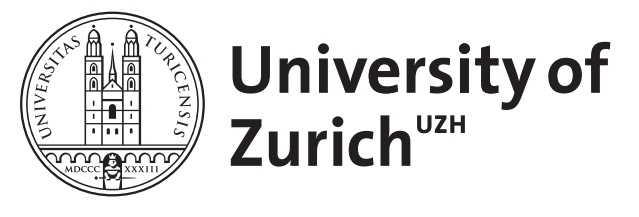

\title{
Exploring number space by random digit generation
}

\author{
Loetscher, Tobias ; Brugger, Peter
}

\begin{abstract}
There is some evidence that human subjects preferentially select small numbers when asked to sample numbers from large intervals "at random". A retrospective analysis of single digit frequencies in 16 independent experiments with the Mental Dice Task (generation of digits 1-6 during 1min) confirmed the occurrence of small-number biases (SNBs) in 488 healthy subjects. A subset of these experiments suggested a spatial nature of this bias in the sense of a "leftward" shift along the number line. First, individual SNBs were correlated with leftward deviations in a number line bisection task (but unrelated to the bisection of physical lines). Second, in 20 men, the magnitude of SNBs significantly correlated with leftward attentional biases in the judgment of chimeric faces. Finally, cognitive activation of the right hemisphere enhanced SNBs in 20 different men, while left hemisphere activation reduced them. Together, these findings provide support for a spatial component in random number generation. Specifically, they allow an interpretation of SNBs in terms of "pseudoneglect in number space." We recommend the use of random digit generation for future explorations of spatial-attentional asymmetries in numerical processing and discuss methodological issues relevant to prospective designs
\end{abstract}

DOI: https://doi.org/10.1007/s00221-007-0889-0

Posted at the Zurich Open Repository and Archive, University of Zurich

ZORA URL: https://doi.org/10.5167/uzh-155919

Journal Article

Published Version

Originally published at:

Loetscher, Tobias; Brugger, Peter (2007). Exploring number space by random digit generation. Experimental Brain Research, 180(4):655-665.

DOI: https://doi.org/10.1007/s00221-007-0889-0 


\title{
Exploring number space by random digit generation
}

\author{
Tobias Loetscher $\cdot$ Peter Brugger
}

Received: 22 September 2006 / Accepted: 16 January 2007 / Published online: 9 February 2007

(c) Springer-Verlag 2007

\begin{abstract}
There is some evidence that human subjects preferentially select small numbers when asked to sample numbers from large intervals "at random". A retrospective analysis of single digit frequencies in 16 independent experiments with the Mental Dice Task (generation of digits 1-6 during $1 \mathrm{~min}$ ) confirmed the occurrence of small-number biases (SNBs) in 488 healthy subjects. A subset of these experiments suggested a spatial nature of this bias in the sense of a "leftward" shift along the number line. First, individual SNBs were correlated with leftward deviations in a number line bisection task (but unrelated to the bisection of physical lines). Second, in 20 men, the magnitude of SNBs significantly correlated with leftward attentional biases in the judgment of chimeric faces. Finally, cognitive activation of the right hemisphere enhanced SNBs in 20 different men, while left hemisphere activation reduced them. Together, these findings provide support for a spatial component in random number generation. Specifically, they allow an interpretation of SNBs in terms of "pseudoneglect in number space." We recommend the use of random digit generation for future explorations of spatial-attentional asymmetries in numerical processing and discuss methodological issues relevant to prospective designs.
\end{abstract}

Keywords Representation of space ·

Random number generation - Pseudoneglect ·

Dual-task

T. Loetscher $(\bowtie) \cdot$ P. Brugger

Neuropsychology Unit, Department of Neurology,

University Hospital Zurich, 8091 Zurich, Switzerland

e-mail: tobias.loetscher@alumni.ethz.ch

\author{
Abbreviations \\ RNG Random number generation \\ MDT Mental dice task \\ SNB Small-number bias \\ LB Line bisection \\ LVF Left visual field \\ RVF Right visual field
}

\section{Introduction}

The last decade has seen an almost exponential increase in the number of publications on conceptual similarities between numerical and spatial processing (see Hubbard et al. 2005 for overview). While early work has pointed to the spatial dimension of simple arithmetic operations (Moyer and Landauer 1967; Restle 1970), the discovery of stimulus response compatibility effects in binary numerical decisions (Dehaene et al. 1993; Fias and Fischer 2005 for review) has stimulated much empirical research on the neural representation of a "number line," extending from left to right in imagined space. A variety of methods converge on the finding that "smaller" numbers are associated with the left-side of space, and "larger" numbers with the right (e.g., Seron et al. 1992; Dehaene et al. 1993; Zorzi et al. 2002; Nuerk et al. 2005).

Some authors have shown a special interest in the interactions between number space and physical space. Fischer, for instance, showed that lines consisting of digits bias observers' bisection marks to the left if the digit is the number 1 , but to the right, if it is number 9 (Fischer 2001). Lines made up by letters representing number words may introduce similar spatial biases (Calabria and Rossetti 2005), and flanker digits to the 
left and right of physical lines may influence subjects' bisections (de Hevia et al. 2006). The speed of manual pointing (Fischer 2003) and the programming of lateral eye-movements (Fischer et al. 2004; Schwarz and Keus 2004) can likewise be influenced by the magnitude of a target digit, small digits favoring leftward and large numbers rightward orienting. A particularly remarkable finding is that detection of left-visual field targets was facilitated after fixation of a small and, vice versa, detection of right-sided targets after a large numeral (Fischer et al. 2003, see Casarotti et al. 2007, for a recent extension of this work).

With respect to clinical work, several studies exploited the left-to-right direction of the number line to show that neglect patients with right parietal lobe damage have an absent or distorted representation of "relatively left-sided," i.e., small compared to large numbers. The methodologies used so far have ranged from magnitude comparisons (Vuilleumier et al. 2004) to assessments of SR-compatibilities (Vuilleumier et al. 2004; Priftis et al. 2006) and explicit bisections of the number line ("What is the median number between 19 and 27?" Zorzi et al. 2002).

In the present study, we aimed at testing the usefulness of yet another method to explore the spatial properties of number space.

This method is briefly sketched in Stanislas Dehaene's popular book "The Number Sense": “...let us pretend that you are a random number generator and that you have to select numbers between 1 and 50 . Once this experiment is performed on a large number of subjects, a systematic bias emerges: instead of responding randomly, we tend to produce smaller numbers more frequently than larger ones - as if smaller numbers were overrepresented in the "mental urn" from which we were drawing." (Dehaene 1997, pp. 76-77). There are in fact empirical data on consistent preferences for small numbers if subjects are required to produce numbers from very large ranges (typically between 1 and infinity or from intervals of "narrow, medium or large," but otherwise unspecified width; Banks and Hill 1974). Originally intended to shed light on the use of numbers as response categories in psychophysical experiments, these studies have contributed significantly to our understanding of the perceived magnitude of numbers and the compression factors of internal numerical scales (see Noma and Baird 1975; Banks and Coleman 1981 for further references). However, to the best of our knowledge, the paradigm of random number generation (RNG) as it is conventionally used in cognitive psychology (Brugger 1997, for a review of some 250 studies) has never been systematically applied to explore the properties of number space. This paradigm requires subjects to draw numbers from a limited, relatively small "mental urn," rarely ever comprising more than the digits 1-10. This omission seems puzzling, as the interpretation of a small-number bias (SNB) as a relative preference for the left over the right side of representational space would seem to have far-reaching consequences for interactions between number processing and spatial cognition (Hubbard et al. 2005). Furthermore, the procedure of generating numbers of a given range "at random" could offer several major advantages over the procedures described above: (1) there is no response effector dichotomy suggestive of the context of "left" and "right" with respect to one's body (as e.g., in the case of manual responses), (2) there are also no obvious conceptual dichotomies that could bias subjects in their assignment of a response category to one side of space (as e.g., in the case of smaller/larger decisions or target numbers belonging to one side of a clockface), and (3) the task is very brief ( $<5 \mathrm{~min})$ and suitable for applications in patient work, as instructions are reportedly easily understood even in the presence of mild to moderate dementia (Brugger et al. 1996). Generally, oral RNG tasks require subjects to repeatedly emit digits at a certain rate and without relying on any rule (Brugger 1997, for overview).

For the present communication, we have reanalyzed the data from various experiments with the Mental Dice Task (MDT), which requires the generation, in one's mind, of 66 consecutive rolls of a die. Study 1 presents a post hoc analysis of 16 experiments with a total of 488 healthy volunteers. Study 2 compared smallnumber preferences in the MDT with subject's performance in a spatial numerical task and study 3 with hemispatial asymmetries in physical space. Finally, we explored whether experimental manipulations known to bias an individual's hemispatial attention in real space would also bias digit preferences in the MDT (study 4).

\section{Study 1: small-number preferences in RNG: a retrospective analysis of healthy subjects' performance on the Mental Dice Task}

Over the past 15 years, various experiments using the MDT paradigm have been carried out in our lab. At the time of running these experiments, we had not been interested in the frequencies of the single digits. However, for most datasets, the respective data were available for the present reanalysis. Specifically, we established whether the frequencies of the digits from 1 
to 6 were randomly distributed or whether there was in fact a systematic bias toward the "small" digits 1, 2, and 3 , as anecdotally described by Dehaene (1997) for a fictitious human random number generator.

\section{Methods}

\section{Subjects}

A total of 488 subjects ( 257 women and 231 men) participated in 16 different experiments (Table 1). The subjects' mean age was 34.9 years (SD 16.6 years) and about half of them were college or university students. A prior history of neurological and psychiatric diseases, of learning disorders, and substance abuse was excluded either by a standardized neuropsychiatric interview adapted from Campbell (2000) or by an abbreviated version thereof. Subjects were righthanded according to published scales or, in experiments 3, 9, and 11, by self-report. Handedness data were lacking for experiments 1,7 , and 16. All testing was undertaken in accordance with the Declaration of Helsinki. Except for the internship projects, each single experiment had been approved by a local Ethics Committee, and all subjects gave written informed consent for participating.

\section{RNG task}

In all 16 experiments, subjects had been administered the MDT. They had thus "to name the digits from 1 to 6 in a sequence as random as possible, i.e., as they might appear when rolling a real die over and over again," In all instances, 66 responses were collected. Generation was usually paced to the beats of a metronome, and a $1 \mathrm{~Hz}$ rhythm was the rule $(1.2 \mathrm{~Hz}$ rhythm in experiments 3,8 , and $14 ; 0.5 \mathrm{~Hz}$ rhythm in experiment 2). Experiments 12, 13, and 15 used unpaced responding. Some of the experiments have been published, and some others approved as diploma theses. Still others were conducted in partial fulfillment of a research internship (see legend to Table 1). Four datasets were collected with native English speaking subjects; all other experiments were performed with subjects with German as the first language.

\section{Results}

On average, 33.7 (SD 2.7) "small" digits (1, 2, or 3) were produced during the generation of 66 digits. This is significantly more than 33.0 small digits expected for a real die $(t=5.50 ; P<0.0001)$. Numerically, there was a surplus of small digits in all 16 experiments (Table 1 ).

Table 1 List of all experiments included for the reanalysis

\begin{tabular}{|c|c|c|c|c|}
\hline Experiment & Language & $\begin{array}{l}\text { Number of subjects } \\
\text { (female/male) }\end{array}$ & $\begin{array}{l}\text { Mean surplus of small } \\
\text { digits over expected } 33\end{array}$ & $\begin{array}{l}P \text {-value } \\
\text { (two-tailed) }\end{array}$ \\
\hline 1 & German & $80(66 / 14)$ & +0.3 & 0.30 \\
\hline 2 & German & $50(28 / 22)$ & +0.7 & $0.03 *$ \\
\hline 3 & German & $43(21 / 22)$ & +0.7 & $0.05^{*}$ \\
\hline 4 & English & $40(20 / 20)$ & +0.5 & 0.58 \\
\hline 5 & German & $39(22 / 17)$ & +1.3 & $0.00 * *$ \\
\hline 6 & German & $34(19 / 15)$ & +0.1 & 0.72 \\
\hline 7 & English & $30(18 / 12)$ & +0.6 & 0.24 \\
\hline 8 & German & $30(-/ 30)$ & +0.4 & 0.47 \\
\hline 9 & English & $25(12 / 13)$ & +0.7 & 0.33 \\
\hline 10 & German & $20(20 /-)$ & +0.9 & 0.12 \\
\hline 11 & English & $20(-/ 20)$ & +1.2 & $0.04 *$ \\
\hline 12 & German & $18(15 / 3)$ & +0.7 & 0.40 \\
\hline 13 & German & $18(8 / 10)$ & +1.5 & $0.04 *$ \\
\hline 14 & German & $18(-/ 18)$ & +0.6 & 0.55 \\
\hline 15 & German & $13(8 / 5)$ & +1.2 & $0.04 *$ \\
\hline 16 & German & $10(-/ 10)$ & +1.4 & 0.12 \\
\hline Total & & $488(257 / 231)$ & +0.68 & $0.00 * *$ \\
\hline
\end{tabular}

For each experiment, the language of generation, the number of subjects tested, and the mean surplus of small $(1,2$ or 3$)$ digits over the expected 33 digits is given. The significance level (one-sample $t$-test) of this surplus is presented in the last column

References: Experiment (Exp.) 1: Brugger et al. (1995); Exp. 4: Brugger et al. under review; Exp. 7: Brugger et al. (1996); Exp. 10: Brugger et al. (1993); Exp. 11: Brugger et al. poster presentation at the Mid-Year Meeting of the International Neuropsychological Society Zurich (2006); Exp. 14: Knoch et al. (2005); Exp. 16: Schütze et al. (1993); Exps. 5, 6, 8, and 12 are unpublished diploma theses. Exps. 2, $3,9,13$, and 15 are unpublished student research projects

$* P \leq 0.05$

$* * P<0.001$ 
In six of them, the small-number preference was independently significant. Of the 488 subjects, 264 (54\%) significantly preferred small digits, and only $151(31 \%)$ had a preference for larger digits $\left(\chi^{2}=30.7\right.$, $P<0.0001)$.

The size of the small-number preference was not modulated by language $(t=0.09, P=0.92)$. There was a tendency for men to name more small-numbers than women $(t=1.47, P=0.14)$.

Figure 1 shows the average frequencies of the single digits in the combined data. Compared to a real die (expected frequency of each digit in 66 rolls $=11.0$ ), the frequency of the digit 5 was significantly underrepresented; digits 2 and 3 were significantly overrepresented.

\section{Brief discussion}

Study 1 unequivocally supports the anecdotal evidence for a preference for small-numbers in human subjects' attempts at naming digits in a random, haphazard way (Dehaene 1997). The bias is small, but its direction is consistent. We found it in each of the 16 experiments. The observed SNB is consistent with data from previous studies investigating subjects' sampling of random numbers from very large, mostly open-ended scales (e.g., Banks and Hill 1974; Baird and Noma 1975). It is also comparable to the data reported by Rath (1966; Fig. 1), who had subjects generate random numbers from the fixed interval between 0 and 9 (each of 20 subjects had to generate 2,500 numbers). Both Rath's and our own findings are in contrast with those of Banks and Coleman (1981; Experiment 6), according to which SNBs occur for unrestricted, but not fixed, sampling

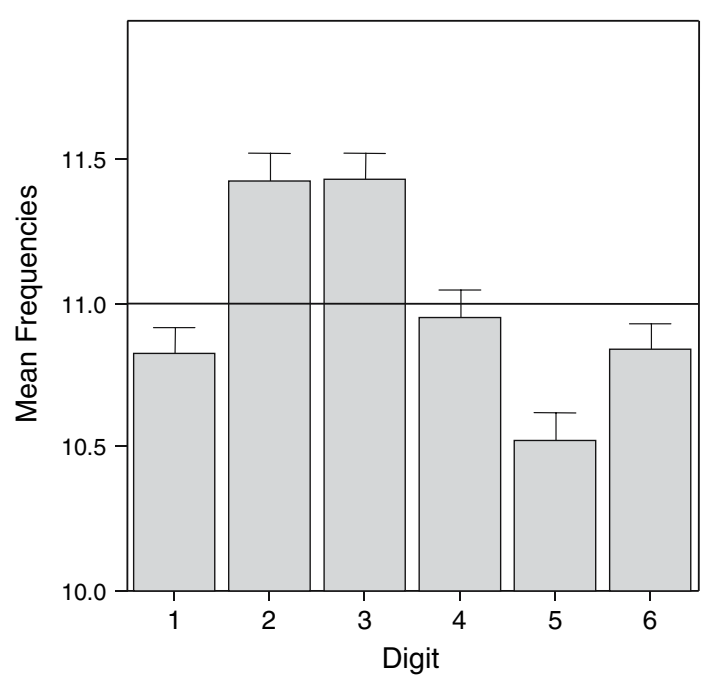

Fig. 1 Average frequencies of the single digits and standard error of mean. Horizontal line denotes the expected frequency (11) for a real die, rolled 66 times ranges. However, these authors have analyzed only ten subject-generated numbers, and future research should examine the extent to which methodological differences in generation rules and number of responses analyzed may be responsible for the discrepancies in the results.

The observed SNB could be the consequence of a compressed internal mental number line, on which the accuracy of mental representations decreases systematically with increasing number size. Such a compression is not necessarily a spatial phenomenon per se. It is reflected, however, in the distance and size effect of numerical processing (Moyer and Landauer 1967; Dehaene 1997). A related observation is that, in everyday language, small digits are more frequently used than large [for example the word "two" is read and heard in English about ten times more often than the word "nine" (Dehaene and Mehler 1992)]. On either hypothesis, one would expect a continuous decrease in the single digit frequencies from 1 to 6 . This is clearly not the case in our data (Fig. 1); digits 1 and 6 were named with comparable frequencies. While the decreasing digit frequencies from 2 to 5 would be compatible with a compressed internal representation, for digits 1 and 6 processes reflecting the special saliency of end points could have played a role.

In terms of spatial processing, the observed smallnumber preference is equivalent to "pseudoneglect", i.e., an exaggerated attention toward left-sided items (Bowers and Heilman 1980; Jewell and McCourt 2000, for review). In other words, as small numbers are "to the left" of large numbers, a leftward attentional bias would automatically result in a SNB.

It seems most parsimonious to conclude from study 1 that a general population bias for small numbers could be established even for the narrow number range of $1-$ 6 . However, study 1 alone does not justify an unequivocal interpretation in spatial terms. We, therefore, examined the potential role of spatial factors more closely.

\section{Study 2: a study on the relationship between small-number preferences in RNG and "leftward" deviations in number line bisection}

If the small-number preference in RNG is indeed of a spatial nature, it should be related to SNBs in other tasks assessing the spatiality of number space. Such biases have been described for healthy subjects and explicitly interpreted as a phenomenon of pseudoneglect (Oliveri et al. 2004; Gobel et al. 2006). We set out to compare number preferences in the MDT with the performance in a traditional number line bisection task (Zorzi et al. 2002, 2006; Priftis et al. 2006). 


\section{Methods}

For three experiments $(2,13$, and 15) presented in Table 1, data were available on subjects' performance in number line bisection (number LB). We pooled the data of these three experiments and correlated individuals' small-number preference in the MDT with the magnitude of their pseudoneglect in number space as assessed explicitly.

\section{Subjects}

Eighty-one subjects (44 women, 37 men) with a mean age of 34.5 years (SD 18.5 years) participated in this experiment. All subjects were right-handed and without prior history of neurological or psychiatric disease. About half of them were college or university students.

\section{Procedure}

In the number LB task, subjects had to name the median number of orally presented numerical intervals. They were instructed to respond as spontaneously as possible and to refrain from calculating the result. Eleven forward (e.g., 1-9) and 11 backward (e.g., 9-1) one-digit number intervals were presented in a pseudorandomized order. For each subject, a number LB index was calculated: "number of errors with the reported number higher than the true median" minus "number of errors with a reported number smaller than the true median" divided by total number of errors. This quotient was then multiplied by 100 . Thus, a negative index score denotes a SNB, interpreted as a leftward deviation on the mental number line. Furthermore, for every subject, the mean difference over all 22 trials of the reported number minus the true median was calculated (number LB deviation).

The MDT was administered as described in study 1 . In analogy to the number LB index, a MDT index was calculated: "number of large digits" minus "number of small digits" divided by the sum. The quotient was multiplied by 100 . A negative MDT index indicates a SNB. An average deviation was also calculated (MDT deviation) as the difference of the arithmetic mean of all 66 produced digits minus the corresponding theoretical value, i.e., 3.5.

\section{Results}

The preference for small digits was reflected in a MDT index significantly different from zero (mean -2.9 , SD $7.0 ; t=-3.7 ; P<0.001$, two-tailed) and in a significant deviation from the true midpoint (average -0.03 , SD $0.12 ; t=-2.1 ; P=0.038$, two-tailed). A SNB was also found in the number LB task (mean number LB index: -9.8, SD 42.7; $t=-2.1 ; P=0.043$, two-tailed; mean number LB deviation: -0.10 , SD $0.22 ; t=-4.2$; $P<0.001$, two-tailed).

There was a significant correlation between the deviations in RNG and number LB tasks (Pearson $r=0.268, \quad P=0.008$, one-tailed), and between the respective indices (Pearson $r=0.195, P=0.040$, onetailed). However, the linear relationship between the preference for small digits in the MDT and the deviation in the number LB task was only significant for men (Pearson $r=0.330, P=0.023$, one-tailed), but fell short of significance for women (Pearson $r=0.21, P=0.090$, one-tailed). There was also no significant correlation between the MDT and number LB indices for the 44 women (Pearson $r=0.140, P=0.188$, one-tailed), while there was at least a strong tendency (Pearson $r=0.241$, $P=0.076$, one-tailed) for the 37 men.

\section{Brief discussion}

We found a significant correlation between subjects' small-number preferences in the MDT and their tendency to err more frequently to the left on the number line in a classical number LB task involving single-digit numbers. To our knowledge, this is the first empirical support for the role of spatial attention in the emergence of RNG. We note that the statistical association between the two kinds of biases is rather modest, yet this may not be surprising if one considers that traditional number LB require a more or less explicit evocation of the number line, whereas this is not the case for the MDT. Previously reported correlations between explicit and implicit measures of number space are reportedly low or absent (Priftis et al. 2006). For an interpretation of the observed gender differences, see the Brief Discussion of study 3.2, below.

\section{Study 3: two studies on the relationships between small-number preferences in RNG and hemispatial biases in physical space}

In order to further investigate the "spatiality" of number space, we compared an individual subject's lateral bias in a visuo-motor and a visual-spatial task with his or her digit preferences (small versus large) in randomizing. Study 3.1 correlated small-number preferences in the MDT with the lateral displacement of the subjective midpoint in the bisection of horizontal lines. Study 3.2 examined the relation between pseudoneglect in number space and a left-sided attention bias in the judgment of the emotional expression of chimeric 
faces. This latter task has been successfully applied for investigations of a variety of individual differences in the strength of subjects' lateral biases (e.g., Luh et al. 1991; Luh and Gooding 1999).

\section{Study 3.1: RNG and line bisection}

This study presents a retrospective analysis of RNG performance as a function of the physical bisection of horizontal lines. Specifically, we were interested whether SNBs in the MDT were statistically associated with the deviations observed in traditional LB tasks. We tested this with the pooled data of experiments 2, 12, 13, and 15 (Table 1), which had required subjects to perform the MDT and a LB task.

\section{Methods}

Subjects Ninety-nine subjects (59 women, 40 men) with a mean age of 34.6 (SD 17.6 years) participated. All subjects were right-handed and without prior history of neurological or psychiatric disease.

Procedure Depending on the experiments, subjects had to bisect either 5 (Experiment 13), 6 (Experiment 12), 9 (Experiment 15), or 10 (Experiment 2) horizontal lines. Line length ranged from 16 to $32 \mathrm{~cm}$ and was not necessarily constant within a given experiment. Each test sheet was centered on the subject's midsagittal plane, and an equal number of lines were displayed to the left on the right side of the sheet. All bisections were performed with the right, dominant hand. For each subject a LB index was calculated: "Number of lines bisected to the right" minus "number of lines bisected to the left" divided by the sum of these terms and multiplied by 100 . Thus, a negative LB index score indicates as leftward deviation and a positive score a rightward deviation from a line's objective midpoint.

The MDT was administered in the standard version described in study 1 . In analogy to the LB index, a MDT index was calculated: "Number of large digits" minus "number of small digits" divided by the sum and multiplied by 100 . A negative MDT index score indicates a SNB, that is, a leftward deviation on the mental number line.

\section{Results}

On average, 33.9 (SD 2.5) small-numbers were produced in the MDT, significantly more than the expected 33 of a real die $(t=3.6, P<0.001)$. There was a significant deviation to the left in the LB task (mean LB index: -14.4 , SD $67.8 ; t=-2.01 ; P=0.038)$.
However, there was neither a correlation between the LB index and MDT index for the whole sample (Pearson $r=0.07, P=0.88$ ), nor separately for the men (Pearson $r=0.18, P=0.27$ ) or the women (Pearson $r=0.16, P=0.22)$.

\section{Brief discussion}

No evidence was found for a relationship between the deviation in LB and number preferences in RNG. This result is in line with Doricchi et al. (2005), who described dissociation, in patients with hemispatial neglect, between physical LB and number LB.

Study 3.2: RNG and perceptual biases in a chimeric faces task

In the experiment reported by Brugger et al. (under review; experiment 4 in Table 1), subjects had been administered, apart from the MDT, a tachistoscopic task on the judgment of the emotional expression of chimeric faces. Here, we were interested in a statistical association between SNB and the amount of left-sided cueing, regularly observed in tasks with chimeric face stimuli, albeit with a high inter-subject variance (Borod et al. 1997).

\section{Methods}

Subjects Twenty women and 20 men participated. They were students of the University of Victoria, Canada, and their mean age was 21.7 years (SD 2.8 years). All subjects were right-handed and without prior history of neurological or psychiatric disease.

Procedure The divided-visual field task comprised a total of 128 trials. Stimuli were 64 line drawings of chimeric faces displaying a sad expression on one side and a happy expression on the other. In $50 \%$ of trials, the happy half-face was presented to the left of the sad half-face; in the remaining $50 \%$, this arrangement was reversed. In 64 trials, only one half-face was presented, either happy or sad and either to the left visual field (LVF) or the right visual field (RVF). Stimuli were presented using an Apple Macintosh computer and the software "Psychlab" (Bub and Gum 1990). Exposure time was $17 \mathrm{~ms}$, and chimeric stimuli extended up to $9.0^{\circ}$ of visual angle to both sides of a central fixation cross. In one run (64 trials), participants pressed the space bar with the index fingers of both hands as soon as they detected a happy half-face or if they considered the chimeric face to look happy rather than sad. In a second run with identical stimuli, they responded to 
sad half-faces or sad-looking chimeric faces, respectively. In both runs 1,500 ms were allotted for a manual response to a trial to be considered and, accordingly, participants were encouraged to decide as spontaneously and fast as possible. The order of the two runs was counterbalanced across subjects. The variable of interest was the total number of trials in which subjects were cued by the LVF part of a chimeric face relative to the number of trials in which they were cued by the RVF part

The MDT was administered in the standard version described in study 1 . We calculated a small-digit ratio by dividing the number of small digits by that of large digits. This index was chosen to make it most analogous to the ratio index used for the chimeric face judgments. In this latter task, subjects widely differed in the number of responses to chimeric faces, and a difference score appeared inappropriate.

\section{Results}

On average, both the 20 men and the 20 women produced 33.5 small digits in the MDT (SD 3.4 for the men, SD 3.6 for the women). This value is not significantly different from the number expected in 66 consecutive rolls of a real die (i.e., 33.0; $t=0.6, P=0.58$ ). Judged emotional facial expression of the 64 chimeric faces was more often cued by the left half-face (19.8 trials, SD 10.1) than by the right half-face (14.9 trials SD 9.7; $t=2.1$, two-tailed $P=0.042$ ). This left-sided bias was comparable for women and men $(t=0.26$, twotailed $P=0.79)$. It was tendentially correlated with the small-digit ratio in the MDT for the 20 men (Pearson $r=0.41, P<0.05$, one-tailed), but not for the 20 women ( $r=0.04, P=0.43$, one-tailed) nor for the sample as a whole $(r=0.23, P=0.08$, one-tailed $)$.

\section{Brief discussion}

The finding in this experiment supports the view that, at least in men, the magnitude of an individual's orienting bias in physical space is related, albeit weakly, to the magnitude of his orienting bias in number space. As in study 2, no comparable relationship was found for the 20 women. This differential result for women and men is in line with recent work reporting gender differences in numerical and spatial processing (Bull and Benson 2006). In that study, more men than women evidenced a spatial strategy in a simple number decision task. If in the present study men attributed more of a spatial character to RNG than women did, a differential pattern of correlation with lateral biases in number LB and the chimeric faces task would not be surprising. Nevertheless, we must admit that the ultimate nature of the observed gender effect is unknown, but according to a recent authoritative review we should be wary of neglecting it altogether (Cahill 2006).

\section{Study 4: a study on the manipulation of small-number preferences in RNG}

Here, we explored whether experimental manipulations known to bias an individual's hemispatial attention in real space would also bias subjects' preference for small or large digits in the MDT. We tested the activation-orientation hypothesis of pseudoneglect (Reuter-Lorenz et al. 1990; Bultitude and Aimola Davies 2006). This hypothesis predicts that a taskinduced increase in hemispheric activation shifts spatial attention contralaterally. We thus analyzed smallnumber preferences in randomization performance during verbal and figural fluency (Experiment 11 in Table 1).

Methods

\section{Subjects}

These were 20 men with a mean age of 38.7 years (SD 14.6 years) and a mean year of education of 14.6 years (SD 2.3 years). All were strongly right-handed by selfreport and worked as hospital staff at the Veterans Administration Hospital San Diego. None had ever suffered from any neurologically or psychiatrically relevant illness.

\section{Procedure}

All subjects first performed the MDT in the standard version, producing a digit between 1 and 6 every second (paced with a metronome). Immediately afterwards, half the subjects repeated this task while simultaneously performing a verbal fluency task, the other half while simultaneously performing a figural fluency task. Concretely, verbal fluency required the generation of as many words as possible that begin with the letter "M," without repetitions or proper names. Words had to be written down, one word per line on a sheet of paper. Figural fluency required the production of as many figures as possible, without repetitions, by connecting five dots by one or more straight lines (Regard et al. 1982). After this second run, subjects once again performed the MDT, this time while simultaneously producing figures (those who 
were first administered the verbal fluency task) or words, respectively. The total number of small digits named was recorded for each run separately.

\section{Results}

Mean number of small digits $(1,2$, and 3$)$ out of the 66 produced numbers was 34.2 (SD 2.4) in the baseline condition, 32.7 (SD 3.4) during verbal fluency, and 36.3 (SD 4.4) during figural fluency (Fig. 2). A one-way ANOVA of these values revealed a significant effect of condition $(F 1,19=12.1, P<0.0001)$. Paired $t$-tests revealed that there were fewer small numbers in the verbal fluency condition than in the baseline condition $(t=2.3$, two-tailed $P=0.033)$, but more in the figural fluency condition ( $t=3.3$, two-tailed $P=0.004)$.

\section{Brief discussion}

The findings of this dual-task experiment combining RNG once with a verbal, left hemisphere-mediated fluency task, and once with figural fluency, reportedly recruiting function of right anterior cortex (Lee et al. 1997), were clear-cut. Compared to a baseline condition, the small-number preference was higher during right anterior activation, but less pronounced during left anterior activity. Although early dual-task experiments on cognitive-motor interactions showed a deterioration of right hand performance by simultaneous verbal activity (Kinsbourne and Cook 1971), the activation-orientation hypothesis of pseudoneglect (Reuter-Lorenz et al. 1990) predicts enhanced attention allocation toward the side opposite the cognitively activated hemisphere. Thus, we interprete the exaggerated SNB in RNG during figural fluency as a task-driven increase in leftward orienting in representational space

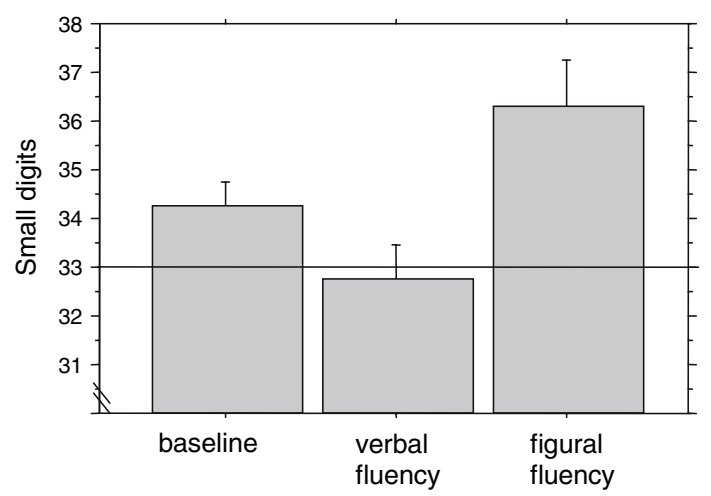

Fig. 2 Number of "small" digits (1, 2, and 3) in the Mental Dice Task during baseline condition and during a simultaneous verbal and figural fluency task $(* P \leq 0.05, * *$ at $P<0.1, N S$ not significantly different to the number expected by chance) (cf. Bultitude and Aimola Davies 2006). We note that the increase in "pseudoneglect in number space" by right hemisphere engagement was larger than the decrease induced by the left hemisphere task. This is in accordance with results of previous studies trying to shift subjects' covert hemispatial attention (Shuren et al. 1998; Gitelman et al. 1999) or to influence hemispheric asymmetries by unilateral vestibular stimulation (Schueli et al. 1999).

\section{General discussion}

The purpose of this study was to test the usefulness of a RNG task to explore spatial properties of "number space". We have reanalyzed data from various experiments carried out in our lab using the MDT, a paradigm which requires the generation, in one's mind, of 66 consecutive rolls of a die (Brugger et al. 1993). The datasets of close to 500 subjects revealed a significant preference for "small" (1-3) over "larger" digits (4-6), an observation that is consistent with Dehaene's (1997) anecdotal proposition of a systematic bias in RNG experiments.

The cause for this bias is not clear. One simple explanation could be that smaller numbers are simply overrepresented because they are used more frequently in everyday life and have a more accurate neural representation on a compressed mental number line (Dehaene and Mehler 1992; Dehaene 1997). Alternatively, also developmental, social-psychological, or linguistic reasons might have contributed to the SNB (see Dehaene and Mehler 1992 for a discussion of some of these aspects in the context of number word frequencies).

Not mutually exclusive to these non-spatial factors is an alternative interpretation that embraces the assumption of "pseudoneglect in number space" (Oliveri et al. 2004; Gobel et al. 2006). In physical space, "pseudoneglect" refers to the observation that healthy subjects tend to bisect lines to the left of the objective midpoint. This right-sided inattention (notably equivalent to a left-sided hyperattention) appears to be universal (Diekamp et al. 2005), is commonly explained in terms of higher-order attentional asymmetries (McCourt and Jewell 1999; Nicholls and Roberts 2002), and known for a considerable between-study variability (Jewell and McCourt 2000, see Table 1 for a comparable variability in the experiments reanalyzed here). Similar mechanisms could underlie asymmetric explorations of representational, especially number space. In other words, as small numbers are "to the left" of large numbers, a leftward attentional bias would automatically 
result in a SNB. The role of spatial attention for the performance in different number tasks has already been demonstrated in neglect patients (Zorzi et al. 2002; Rossetti et al. 2004) as well as in healthy subjects (Fischer 2001; Fischer et al. 2003). Several neuroimaging studies (see Dehaene et al. 2003) converge in the finding that circuits in the posterior superior parietal lobes mediate the orientation along the number line as much as they subserve attentional orientation in physical space. Interestingly, similar activation loci were described in neuroimaging experiments of RNG (Jahanshahi et al. 2000; Daniels et al. 2003). Thus, we went on to investigate the influence of spatial factors on RNG more carefully. Specifically, we examined potential associations of small-number preferences in RNG with other forms of lateralized behavior.

In study 2 , we found a correlation between the magnitude of an individual's small-number preference in RNG and the magnitude of his or her "leftward" bias in a number bisection task. As emphasized by Priftis et al. (2006, p. 681), this task requires "voluntary access of the spatial frame of the mental number line, presumably through the orientation of spatial attention." Thus, the correlation of a randomization bias in favor of small digits and the small-number deviation in a task assessing the spatial frame of the mental number line suggests that spatial attentional factors may guide subject's behavior in both tasks. If solely non-spatial factors accounted for the weak but consistent bias observed in study 1 , one would hardly expect a correlation between these two tasks.

After having gathered some evidence for spatial factors contributing to the small-number preference in representational space, we compared the relations of this bias to hemispatial asymmetries in physical space. While the SNB was unrelated to simple LB performance, we found it positively correlated to the magnitude of left-sided cueing in a task requiring the judgment of chimeric faces (for male, but not female participants). A dissociation between traditional LB and number LB has already been reported by Doricchi et al. (2005) in neglect patients, and the absence of a significant correlation between the two deviations, one in physical space and notably requiring a motor response, and the other in number space, may not be surprising. The correlation between the SNB and asymmetric judgments of facial expressions is remarkable, however modest in magnitude and even if it was confined to the $20 \mathrm{men}$. If confirmed in prospective studies, it would indicate that posterior parietal attention systems are more important for directing one's attention in number space than are more anterior, action-related systems (see however, Doricchi et al.
2005). The absence of a correlation in our female participants may be explained by pointing out women's more analytic, less space-related style of processing (Tranel et al. 2005).

Most promising was our evaluation of whether manipulations of spatial attention can alter the smallnumber preferences in RNG. According to the activation-orientation hypothesis, spatial attention is biased in a direction away from the more activated hemisphere (Reuter-Lorenz et al. 1990; Bultitude and Aimola Davies 2006). We used two cognitive tasks with known recruitment demands of left or right hemisphere functions. During a written verbal fluency task (left hemisphere mediated), the SNB in the simultaneously performed MDT was diminished, while a figural fluency task (right hemisphere mediated) enhanced it. A task-driven increase in right hemisphere activation thus led to a contralateral allocation of attention in number space.

To summarize, we note that effect sizes in the present communication are rather small, both in terms of the difference between small and large digits and in the correlations of the observed small-number preferences and various measures of hemispatial attention. It is the consistency of the findings across the different experiments and correlates of spatial processing that makes us trust in a genuinely spatial component of SNBs.

The series of experiments described in the present paper show both strengths and weaknesses of any retrospective analysis. Among the strengths is certainly the fact that the findings cannot be attributed to expectation effects or experimenter biases. At the time of running the single experiments, we were not concerned with "asymmetries in number space" and were unaware of the meaningfulness of any analysis regarding single digit frequencies. As we analyzed all studies whose data were available, we need not be concerned about any serious selection bias either. On the other hand, these very strengths can also be considered a major weakness of our approach: as we had selected study settings according to the original research questions (always concerned with response habits of a sequential nature; Brugger 1997 for overview), the experimental designs were not optimally adapted to the exploration of the spatial characteristics of numerical intervals. Another shortcoming arises from to the particular RNG task we used. It is the restriction to the digits from 1 to 6 . Clearly, this number range is much too narrow to draw definite conclusions about spatial aspects of number processing in general. Future studies should investigate small-number preferences in RNG across larger numerical intervals and also directly examine the effect of interval size on the magnitude of 
"pseudoneglect" with special attention to the issue of a cross-over effect in number space (see Rueckert et al. 2002 for such an effect in physical space and Zorzi et al. 2006 for cross-over in number space). Our retrospective analyses must also leave open the question concerning the relationships between the compression factor of a particular numerical interval as determined by number production methods used by Banks and Hill (1974) and the observed SNB if subjects sample from the same interval but with more standard, paced randomization instructions. Furthermore, the spatial nature of SNBs in RNG should be explored by correlational analyses comparing an individual's preference for small numbers with his or her size and distance effects in standard numerical decision tasks.

Finally, our present reanalyses are based solely on RNG by healthy subjects. Studies of small-number preferences in patients with neglect (notably comprising the representation of space) should be on the top of the research agenda.

Keeping in mind the caveats discussed above, the present study provides a fair amount of evidence for the usefulness of RNG as a method to explore spatial properties of number space, especially in combination with manipulations known to bias subject's attention in physical space. Should prospective studies by independent research groups confirm this assumption, it would be particularly interesting to learn about how spatial (i.e., posterior parietal) and non-spatial (i.e., frontalexecutive) components of RNG interact with one another. By this, we could gain insight not only into the spatial properties of numerical cognition, but also into the sequential planning aspects of spatial orientation.

Acknowledgments We thank the investigators of the original experiments on RNG for having administered the Mental Dice Task. We also acknowledge financial support by the Betty and David Koetser foundation (to PB).

\section{References}

Baird JC, Noma E (1975) Psychophysical study of numbers I. Generation of numerical responses. Psychol Res Psychol Forsch 37:281-297

Banks WP, Coleman MJ (1981) Two subjective scales of number. Percept Psychophys 29:95-105

Banks WP, Hill DK (1974) Apparent magnitude of number scaled by random production. J Exp Psychol 102:353-376

Borod JC, Haywood CS, Koff E (1997) Neuropsychological aspects of facial asymmetry during emotional expression: a review of the normal adult literature. Neuropsychol Rev 7:41-60

Bowers D, Heilman KM (1980) Pseudoneglect-effects of hemispace on a tactile line bisection task. Neuropsychologia 18:491-498

Brugger P (1997) Variables that influence the generation of random sequences: an update. Percept Mot Skills 84:627-661
Brugger P, Milicevic A, Regard M, Cook ND (1993) Randomnumber generation and the menstrual-cycle-preliminary evidence for a premenstrual alteration of frontal-lobe functioning. Percept Mot Skills 77:915-921

Brugger P, Monsch AU, Salmon DP, Butters N (1996) Random number generation in dementia of the Alzheimer type: a test of frontal executive functions. Neuropsychologia 34:97-103

Brugger P, Pietzsch S, Weidmann G, Biro P, Alon E (1995) Stroop-type interference in random-number generation. Psychol Rep 77:387-390

Bub D, Gum T (1990) Psychlab. In: McGill Technical Manuals, McGill University, Montreal

Bull R, Benson PJ (2006) Digit ratio (2D: 4D) and the spatial representation of magnitude. Horm Behav 50:194-199

Bultitude JH, Aimola Davies AM (2006) Putting attention on the line: investigating the activation-orientation hypothesis of pseudoneglect. Neuropsychologia 44:1849-1858

Cahill L (2006) Why sex matters for neuroscience. Nat Rev Neurosci 7:477-484

Calabria M, Rossetti Y (2005) Interference between number processing and line bisection: a methodology. Neuropsychologia 43:779-783

Campbell JJ (2000) Neuropsychiatric assessment. In: Coffey CE, Cummings JL (eds) Textbook of Geriatric Neuropsychiatry. American Psychiatric Publishing, Washington, DC, pp 109-124

Casarotti M, Michielin M, Zorzi M, Umilta C (2007) Temporal order judgment reveals how number magnitude affects visuospatial attention. Cognition 102:101-117

Daniels C, Witt K, Wolff S, Jansen O, Deuschl G (2003) Rate dependency of the human cortical network subserving executive functions during generation of random number series-a functional magnetic resonance imaging study. Neurosci Lett 345:25-28

de Hevia MD, Girelli L, Vallar G (2006) Numbers and space: a cognitive illusion? Exp Brain Res 168:254-264

Dehaene S (1997) The number sense. How the mind creates mathematics. Oxford University Press, Oxford

Dehaene S, Bossini S, Giraux P (1993) The mental representation of parity and number magnitude. J Exp Psychol Gen 122:371-396

Dehaene S, Mehler J (1992) Cross-linguistic regularities in the frequency of number words. Cognition 43:1-29

Dehaene S, Piazza M, Pinel P, Cohen L (2003) Three parietal circuits for number processing. Cogn Neuropsychol 20:487-506

Diekamp B, Regolin L, Gunturkun O, Vallortigara G (2005) A left-sided visuospatial bias in birds. Curr Biol 15:R372-R373

Doricchi F, Guariglia P, Gasparini M, Tomaiuolo F (2005) Dissociation between physical and mental number line bisection in right hemisphere brain damage. Nat Neurosci 8:1663-1665

Fias W, Fischer MH (2005) Spatial representation of numbers. In: Campbell JID (eds) Handbook of mathematical cognition. Psychology Press, New York, pp 43-54

Fischer MH (2001) Number processing induces spatial performance biases. Neurology 57:822-826

Fischer MH (2003) Spatial representations in number processing-evidence from a pointing task. Vis Cogn 10:493-508

Fischer MH, Castel AD, Dodd MD, Pratt J (2003) Perceiving numbers causes spatial shifts of attention. Nat Neurosci 6:555-556

Fischer MH, Warlop N, Hill RL, Fias W (2004) Oculomotor bias induced by number perception. Exp Psychol 51:91-97

Gitelman DR, Nobre AC, Parrish TB, LaBar KS, Kim YH, Meyer JR, Mesulam M (1999) A large-scale distributed network for covert spatial attention: further anatomical delineation based on stringent behavioural and cognitive controls. Brain 122:1093-1106 
Gobel SM, Calabria M, Farne A, Rossetti Y (2006) Parietal rTMS distorts the mental number line: Simulating "spatial" neglect in healthy subjects. Neuropsychologia 44:860-868

Hubbard EM, Piazza M, Pinel P, Dehaene S (2005) Interactions between number and space in parietal cortex. Nat Rev Neurosci 6:435-448

Jahanshahi M, Dirnberger G, Fuller R, Frith CD (2000) The role of the dorsolateral prefrontal cortex in random number generation: a study with positron emission tomography. Neuroimage 12:713-725

Jewell G, McCourt ME (2000) Pseudoneglect: a review and metaanalysis of performance factors in line bisection tasks. Neuropsychologia 38:93-110

Kinsbourne M, Cook J (1971) Generalized and lateralized effects of concurrent verbalization on a unimanual skill. Q J Exp Psychol 23:341-345

Knoch D, Brugger P, Regard M (2005) Suppressing versus releasing a habit: frequency-dependent effects of prefrontal transcranial magnetic stimulation. Cereb Cortex 15:885-887

Lee GP, Struss E, Loring DW, McCloskey L, Haworth JM, Lehman RAW (1997) Sensitivity of figural fluency on the five-point test to focal neurological dysfunction. Clin Neuropsychol 11:59-68

Luh KE, Gooding DC (1999) Perceptual biases in psychosisprone individuals. J Abnorm Psychol 108:283-289

Luh KE, Rueckert LM, Levy J (1991) Perceptual asymmetries for free viewing of several types of chimeric stimuli. Brain Cogn 16:83-103

McCourt ME, Jewell G (1999) Visuospatial attention in line bisection: stimulus modulation of pseudoneglect. Neuropsychologia 37:843-855

Moyer RS, Landauer TK (1967) Time required for judgements of numerical inequality. Nature 215:1519-1520

Nicholls MER, Roberts GR (2002) Can free-viewing perceptual asymmetries be explained by scanning, pre-motor or attentional biases? Cortex 38:113-136

Noma E, Baird JC (1975) Psychophysical study of numbers II. Theoretical models of number generation. Psychol Res Psychol Forsch 38:81-95

Nuerk HC, Wood G, Willmes K (2005) The universal SNARC effect-the association between number magnitude and space is amodal. Exp Psychol 52:187-194

Oliveri M, Rausei V, Koch G, Torriero S, Turriziani P, Caltagirone $\mathrm{C}$ (2004) Overestimation of numerical distances in the left side of space. Neurology 63:2139-2141

Priftis K, Zorzi M, Meneghello F, Marenzi R, Umilta C (2006) Explicit versus implicit processing of representational space in neglect: dissociations in accessing the mental number line. J Cogn Neurosci 18:680-688

Rath G (1966) Randomization by humans. Am J Psychol 79:97103

Regard M, Strauss E, Knapp P (1982) Children's production on verbal and non-verbal fluency tasks. Percept Mot Skills $55: 839-844$

Restle F (1970) Speed of adding and comparing numbers. J Exp Psychol 83:274-278

Reuter-Lorenz PA, Kinsbourne M, Moscovitch M (1990) Hemispheric control of spatial attention. Brain Cogn 12:240-266

Rossetti Y, Jacquin-Courtois S, Rode G, Ota H, Michel C, Boisson D (2004) Does action make the link between number and space representation? Visuo-manual adaptation improves number bisection in unilateral neglect. Psychol Sci 15:426-430

Rueckert L, Deravanesian A, Baboorian D, Lacalamita A, Repplinger M (2002) Pseudoneglect and the cross-over effect. Neuropsychologia 40:162-173

Schueli H, Henn V, Brugger P (1999) Vestibular stimulation affects dichotic lexical decision performance. Neuropsychologia 37:653-659

Schütze H, Bürki P, Brugger P, Koller E (1993) Neuropsychological reactions at moderate altitude. In: Sutton J, Houston C, Coates G (eds) Hypoxia and molecular medicine. Queen City Printers Inc., Burlington, p 313

Schwarz W, Keus IM (2004) Moving the eyes along the mental number line: comparing SNARC effects with saccadic and manual responses. Percept Psychophys 66:651-664

Seron X, Pesenti M, Noel MP, Deloche G, Cornet JA (1992) Images of numbers, or when 98 is upper left and 6 sky blue. Cognition 44:159-196

Shuren J, Hartley T, Heilman KM (1998) The effects of rotation on spatial attention. Neuropsychiatry Neuropsychol Behav Neurol 11:72-75

Tranel D, Damasio H, Denburg NL, Bechara A (2005) Does gender play a role in functional asymmetry of ventromedial prefrontal cortex? Brain 128:2872-2881

Vuilleumier P, Ortigue S, Brugger P (2004) The number space and neglect. Cortex 40:399-410

Zorzi M, Priftis K, Meneghello F, Marenzi R, Umilta C (2006) The spatial representation of numerical and non-numerical sequences: evidence from neglect. Neuropsychologia 44:1061-1067

Zorzi M, Priftis K, Umilta C (2002) Brain damage: neglect disrupts the mental number line. Nature 417:138-139 